Proceedings of the

International Geometry Center

Vol. 10, no. 2 (2017) pp. 56-69

\title{
Объем конечного ортогонального $h$-конуса в гиперболическом пространстве положительной кривизны
}

\author{
Л. Н. Ромакина
}

\begin{abstract}
In the projective Cayley-Klein model the hyperbolic space $\widehat{H}^{3}$ of positive curvature is realized on the ideal domain of the Lobachevskii space. In this work the basic notions of the volumes theory of the space $\widehat{H}^{3}$ are introduced through invariants of the fundamental group of the space. In the orthogonal curvilinear coordinate system the volume formula of a finite orthogonal $h$-cone and some corollaries from it are proved.

Аннотация. В проективной модели Кэли-Клейна гиперболическое пространство $\widehat{H}^{3}$ положительной кривизны реализовано на идеальной области пространства Лобачевского. В работе введены основные понятия теории объемов пространства $\widehat{H}^{3}$ через инварианты фундаментальной группы пространства. В ортогональной криволинейной системе координат доказаны формула объема конечного ортогонального $h$-конуса и некоторые следствия из нее.
\end{abstract}

\section{1. ВВЕДЕНИЕ}

1.1. Гиперболическое пространство положительной кривизны. Гиперболическое пространство $\widehat{H}^{3}$ положительной кривизны [14, с. 210] может быть реализовано на гиперсфере вещественного радиуса с отождествленными диаметрально противоположными точками в псевдоевклидовом пространстве $\mathbb{R}_{1}^{4}$. Но больший интерес, на наш взгляд, представляет интерпретация пространства $\widehat{H}^{3}$ в проективной схеме КэлиКлейна, поскольку она может быть использована для описания взаимодействия атомных частиц [23]. В проективной модели пространство

2010 Mathematics Subject Classification: 51F10, 51M25

Ключевые слова: гиперболическое пространство положительной кривизны, объем ортогонального конуса, объем ортогональной пирамиды

УДК 514.133 
$\widehat{H}^{3}$ реализовано на идеальной области пространства Лобачевского $\Lambda^{3}$. Пространства $\widehat{H}^{3}$ и $\Lambda^{3}$ являются связными компонентами расширенного гиперболического пространства $\mathbb{H}^{3}$, т. е. проективного пространства $\mathbb{P}^{3}$ с бесконечно удаленной овальной поверхностью, называемой абсолютом пространств $\widehat{H}^{3}, \Lambda^{3}$ и $\mathbb{H}^{3}[14]$. Напомним, что овальной поверxностью пространства $\mathbb{P}^{3}$ называют невырожденную поверхность второго порядка сигнатуры 2 [11]. Пространство Лобачевского реализуется на внутренней, а пространство $\widehat{H}^{3}$ - на внешней области пространства $\mathbb{P}^{3}$ относительно абсолюта. Группа $G$ проективных автоморфизмов овальной поверхности является фундаменталъной группой преобразований пространств $\widehat{H}^{3}, \Lambda^{3}$ и $\mathbb{H}^{3}$.

Все прямые пространства $\widehat{H}^{3}$ в зависимости от количества и природы общих с абсолютом точек образуют три типа [22]. Эллиптические ( зиперболические) прямые пересекают абсолют в двух мнимо сопряженных (вещественных) точках. Параболические прямые касаются абсолюта и являются изотропными в пространстве $\widehat{H}^{3}$. С каждой собственной точкой пространства $\widehat{H}^{3}$ связан конус изотропных образующих с вершиной в этой точке, названный изотропным или световым конусом данной точки. Каждая эллиптическая (гиперболическая) прямая, проходящая через собственную точку пространства $\widehat{H}^{3}$, принадлежит внутренней (внешней) области относительно светового конуса данной точки.

Все плоскости пространства $\widehat{H}^{3}$ в зависимости от типа линии пересечения с абсолютом образуют также три типа [22]. Эллиптические плоскости пересекают абсолютную поверхность по нулевой линии [11]. Гиперболические плоскости положительной кривизны (см., например, [17], [18]) имеют с абсолютом общую овальную линию и представлют собой внешние относительно абсолюта компоненты расширенных гиперболических плоскостей. Коевклидовы плоскости (см., например, [9], [15]) пересекают абсолют по вырожденной линии второго порядка паре мнимо сопряженных прямых.

Двугранные углы пространства $\widehat{H}^{3}$ как и углы гиперболической плоскости $\widehat{H}$ положительной кривизны образуют 15 типов, углы шести типов измеримы с помощью абсолюта, углы трех типов имеют вещественные меры [16], [17], [6]. Каждый угол коевклидовой плоскости принадлежит одному из четырех типов, для углов между параболическими прямыми такой плоскости существует инвариант фундаментальной группы преобразований, для конечных углов между эллипическими прямыми существует инвариант группы движений [15]. 
1.2. Постановка задачи. Теория объемов в пространстве Лобачевского с первых своих шагов обогащала математику новыми результатами и новыми методами. Она имеет давнюю историю и остается актуальной на современном этапе (см., например, [13], [10], [25], [8], [1], [2], [3], [4], [7]). Значительные трудности вычисления объемов многогранников в пространстве Лобачевского, образно названные Гауссом «джунгли», на протяжении двух веков поддерживают дух состязательности среди ученых, укрепляя их в стремлении получить наиболее компактные формулы объемов. Но само пространство будто подшучивает над исследователями, оставляя в лидерах первопроходцев и стимулируя тем самым их последователей к выходу на свою идеальную область.

В поисках аналогов простых формул объема из евклидовой и эллиптической геометрий, например, формулы $V=\frac{1}{6} a b c$ объема евклидова тетраэдра с исходящими из одной вершины попарно ортогональными ребрами или формулы $V=\frac{1}{2} a b$ объема ортосферы в эллиптическом пространстве, исследователь непременно задается вопросом об особенностях строения пространства Лобачевского, затрудняющих получение таких формул. Довольно простое объяснение этого вопроса дает проективная модель данного пространства.

Полное пространство $\Lambda^{3}$, реализуясь внутри овальной поверхности, не содержит, в отличие от евклидова или эллиптического пространства, скрещивающихся взаимно ортогональных прямых. Напомним, что ортогональность в проективном смысле означает сопряженность относительно абсолюта. Для каждой прямой плоскости Лобачевского существует пучок ортогональных ей прямых, центр которого расположен в полюсе данной прямой относительно абсолютной овальной линии. Поэтому для каждой прямой пространства Лобачевского поиск ортогональной пересекающейся с ней прямой всегда дает положительный результат. Иначе обстоит дело со скрещивающимися прямыми. Сопряженность относительно абсолюта пространства, теперь уже овальной поверхности, возможна только в парах прямых различных типов. Для каждой прямой пространства Лобачевского, т. е. гиперболической прямой, существует лишь единственная ортогональная скрещивающаяся с ней прямая, причем расположена эта прямая за пределами пространства, является эллиптической. Изучая конечные объекты пространства $\Lambda^{3}$ и используя системы координат в его собственной области, задействовать такую прямую исследователи не могут, следовательно не могут максимально упростить вычисления.

Ситуация меняется принципиально при выходе исследований на идеальную область пространства $\Lambda^{3}$. «Прорыв» за пределы абсолюта, с одной стороны, значительно обогащает гиперболическую геометрию, а с 
другой - позволяет существенно упростить решение некоторых задач. В данной работе представлен пример одной из таких задач - вычисление в пространстве $\widehat{H}^{3}$ объемов конечного ортогонального $h$-конуса и тел, полученных его рассечением эллиптической сферой.

Прежде чем приступить к решению, по схеме, предложенной в работах [20], [24], определим основные понятия теории объемов пространства $\widehat{H}^{3}$ в проективной модели Кэли-Клейна и введем ортогональную криволинейную систему координат с базовой расширенной гиперболической плоскостью, определенную на внешней области относительно светового конуса своего полюса. Применяя введенную систему, установим зависимость объема конечного ортогонального $h$-конуса от площади его основания. Как следствия искомой зависимости получим формулы объема конечной ортогональной пирамиды с гиперболическим основанием.

1.3. Основные метрические формулы. Все вычисления в пространстве $\widehat{H}^{3}$ проводим в каноническом репере $R^{*}=\left\{A_{1}, A_{2}, A_{3}, A_{4}, E\right\}$ первого типа, вершины которого попарно сопряжены относительно абсолюта, вершина $A_{4}$ является внутренней по отношению к абсолюту, а единичная точка $E$ служит пересечением трех коевклидовых плоскостей, каждая из которых содержит одну из координатных прямых $A_{1} A_{2}, A_{1} A_{3}, A_{2} A_{3}$. Семейство всех канонических реперов первого типа в пространстве $\widehat{H}^{3}$ зависит от шести параметров. Уравнение абсолютной поверхности $\gamma$ в каждом репере этого семейства имеет вид

$$
x_{1}^{2}+x_{2}^{2}+x_{3}^{2}-x_{4}^{2}=0 .
$$

Квадратичная форма $\varphi\left(x_{1}, x_{2}, x_{3}, x_{4}\right)=x_{1}^{2}+x_{2}^{2}+x_{3}^{2}-x_{4}^{2}$ является метрической формой в пространстве $\widehat{H}^{3}$. Для собственной (идеальной) точки $A$ пространства $\widehat{H}^{3}$ с координатами $\left(a_{p}\right), p=1,2,3,4$, в репере $R^{*}$ справедливо неравенство

$$
a_{1}^{2}+a_{2}^{2}+a_{3}^{2}-a_{4}^{2}>0 \quad\left(a_{1}^{2}+a_{2}^{2}+a_{3}^{2}-a_{4}^{2}<0\right) .
$$

Если точки $A$ и $B$ эллиптической (гиперболической) прямой заданы в репере $R^{*}$ координатами соответственно $\left(a_{p}\right)$ и $\left(b_{p}\right), p=1,2,3,4$, то расстояние между ними может быть выражено по формуле

$$
\begin{gathered}
\cos \frac{|A B|}{\rho}= \pm \frac{a_{1} b_{1}+a_{2} b_{2}+a_{3} b_{3}-a_{4} b_{4}}{\sqrt{a_{1}^{2}+a_{2}^{2}+a_{3}^{2}-a_{4}^{2}} \sqrt{b_{1}^{2}+b_{2}^{2}+b_{3}^{2}-b_{4}^{2}}} \\
\left(\cosh \frac{|A B|}{\rho}= \pm \frac{a_{1} b_{1}+a_{2} b_{2}+a_{3} b_{3}-a_{4} b_{4}}{\sqrt{a_{1}^{2}+a_{2}^{2}+a_{3}^{2}-a_{4}^{2}} \sqrt{b_{1}^{2}+b_{2}^{2}+b_{3}^{2}-b_{4}^{2}}}\right),
\end{gathered}
$$


где $\rho$ - радиус кривизны пространства $\widehat{H}^{3}, \rho \in \mathbb{R}_{+}$.

Билинейная форма $\bar{\varphi}$, полярная к форме $\varphi$, определяет условие ортогональности точек $A$ и $B$ в репере $R^{*}$ :

$$
a_{1} b_{1}+a_{2} b_{2}+a_{3} b_{3}-a_{4} b_{4}=0 .
$$

Тангенциальная метрическая форма пространства $\widehat{H}^{3}$ в репере $R^{*}$ совпадает с формой $\varphi$. Для координат $\left(\alpha_{p}\right), p=1,2,3,4$, расширенной гиперболической (эллиптической) плоскости $\alpha$ пространства $\widehat{H}^{3}$ в репере $R^{*}$ выполняется неравенство

$$
\alpha_{1}^{2}+\alpha_{2}^{2}+\alpha_{3}^{2}-\alpha_{4}^{2}>0 \quad\left(\alpha_{1}^{2}+\alpha_{2}^{2}+\alpha_{3}^{2}-\alpha_{4}^{2}<0\right) .
$$

2. ОСНОВНЫЕ ПОЛОЖЕНИЯ ТЕОРИИ ОБЪЕМОВ ПРОСТРАНСТВА $\widehat{H}^{3}$

По схеме из работы [24] определим в пространстве $\widehat{H}^{3}$ понятие объема тела через проективные инварианты фундаментальной группы $G$.

2.1. Собственные координаты точек в пространстве $\widehat{H}^{3}$. Проективные координаты точек пространства $\widehat{H}^{3}$ являются однородными, поэтому их использование не позволяет однозначно определить понятие объема тела этого пространства. Чтобы избежать неоднозначности вводимых инвариантов, проведем нормировку проективных координат точек, инвариантную относительно действий группы $G$.

Предположим, что в репере $R^{*}$ вещественные числа $\left(x_{p}\right), p=1,2,3,4$, служат координатами собственной точки $M$ пространства $\widehat{H}^{3}$ радиуса кривизны $\rho, \rho \in \mathbb{R}_{+}$. Тогда четверку чисел

$$
\bar{x}_{p}= \pm \frac{\rho x_{p}}{\sqrt{x_{1}^{2}+x_{2}^{2}+x_{3}^{2}-x_{4}^{4}}},
$$

определенных с точностью до общего ненулевого вещественного множителя, назовем собственными координатами точки $M$ в репере $R^{*}$.

На основании условий (1.1) собственным (идеальным) точкам пространства $\widehat{H}^{3}$ в нормировке (2.1) соответствуют определенные до знака четверки вещественных (мнимых) чисел, а точкам абсолюта - четверки бесконечно больших величин.

Собственные координаты собственных точек пространства $\widehat{H}^{3}$ удовлетворяют равенство

$$
\bar{x}_{1}^{2}+\bar{x}_{2}^{2}+\bar{x}_{3}^{2}-\bar{x}_{4}^{2}=\rho^{2} .
$$


2.2. Элемент объема пространства $\widehat{H}^{3}$. Рассмотрим в пространстве $\widehat{H}^{3}$ область $Q$, гомеоморфную открытому шару (или, на языке проективной геометрии, внутренности овальной поверхности проективного пространства $\mathbb{P}^{3}$ ). Пусть в репере $R^{*}$ произвольная точка $M$ из области $Q$ имеет собственные координаты $\left(\bar{x}_{p}\right)$. Зададим на области $Q$ криволинейную систему координат $C^{*}$ гладкими функциями:

$$
\bar{x}_{p}=\bar{x}_{p}(u, v, w), p=1,2,3,4,(u, v, w) \in \bar{Q} \subset \mathbb{R}^{3} .
$$

Репер $R^{*}$ будем называть присоединенным к системе $C^{*}$.

Условимся, что любые две координатные кривые в каждом семействе $u, v$, или $w$ системы $C^{*}$ принадлежат одному непараболическому типу кривых, определенному типом касательной к кривой в каждой ее точке [24]. Систему $C^{*}$ назовем ортогональной, если в каждой точке области $Q$ координатные кривые попарно ортогональны.

Согласно условиям $(1.4),(2.2)$ точки

$$
M_{u}\left(\frac{\partial \bar{x}_{p}}{\partial u}\right), M_{v}\left(\frac{\partial \bar{x}_{p}}{\partial v}\right), M_{w}\left(\frac{\partial \bar{x}_{p}}{\partial w}\right)
$$

ортогональны точке $M$. Поэтому плоскость $M_{u} M_{v} M_{w}$ - полярная плоскость точки $M$ относительно абсолюта.

Для значений форм $\bar{\varphi}$ и $\varphi$ от координат точек (2.3) введем следующие обозначения:

$$
\begin{gathered}
\gamma_{u v}=\bar{\varphi}\left(\frac{\partial \bar{x}_{p}}{\partial u}, \frac{\partial \bar{x}_{p}}{\partial v}\right), \gamma_{v w}=\bar{\varphi}\left(\frac{\partial \bar{x}_{p}}{\partial v}, \frac{\partial \bar{x}_{p}}{\partial w}\right), \gamma_{u w}=\bar{\varphi}\left(\frac{\partial \bar{x}_{p}}{\partial u}, \frac{\partial \bar{x}_{p}}{\partial w}\right) \\
\gamma_{u u}=\varphi\left(\frac{\partial \bar{x}_{p}}{\partial u}\right), \gamma_{v v}=\varphi\left(\frac{\partial \bar{x}_{p}}{\partial v}\right), \gamma_{w w}=\varphi\left(\frac{\partial \bar{x}_{p}}{\partial w}\right) .
\end{gathered}
$$

В системе $C^{*}$ прямые $M M_{u}, M M_{v}$ и $M M_{w}$ являются касательными к координатным линиям $u, v$ и, соответственно, $w$ в точке $M$. Следовательно, ортогональность системы $C^{*}$ равносильна системе условий:

$$
\gamma_{u v}=\gamma_{v w}=\gamma_{u w}=0
$$

Непосредственная проверка показывает, что число

$$
J=\left|\begin{array}{lll}
\gamma_{u u} & \gamma_{u v} & \gamma_{u w} \\
\gamma_{u v} & \gamma_{v v} & \gamma_{v w} \\
\gamma_{u w} & \gamma_{v w} & \gamma_{w w}
\end{array}\right|
$$


удовлетворяет равенство $J=-\Phi$, где $\Phi-$ значение метрической формы $\varphi$ от координат плоскости $M_{u} M_{v} M_{w}$ в репере $R^{*}$. Следовательно, число $J$ - инвариант группы $G$, характеризующий сопровождающий тетраэдр $M M_{u} M_{v} M_{w}$ точки $M$. Поскольку $M$ - собственная точка пространства $\widehat{H}^{3}$, ее абсолютная полярная плоскость $M_{u} M_{v} M_{w}$ является расширенной гиперболической. Согласно условию (1.5) для плоскости $M_{u} M_{v} M_{w}$ справедливо неравенство $\Phi>0$. При измерении объемов конечных тел мы стремимся использовать вещественные положительные числа, поэтому в качестве элемента объема пространства $\widehat{H}^{3}$ примем число

$$
d V=\sqrt{-J} d u d v d w .
$$

2.3. Формула объема тела в пространстве $\widehat{H}^{3}$. Зададим в области $Q$ пространства $\widehat{H}^{3}$ конечное тело $F$, гомеоморфное замыканию внутренности овальной поверхности пространства $\mathbb{P}^{3}$. Условимся, что числовая область $\bar{F}$, где $\bar{F} \subset \bar{Q} \subset \mathbb{R}^{3}$, определяет тело $F$ в координатной системе $C^{*}$ на области $Q$. Тогда согласно формуле $(2.4)$ объем $V$ тела $F$ может быть выражен по формуле

$$
V=\iiint_{\bar{F}} \sqrt{-J} d u d v d w .
$$

Заметим, что при вычислении по формуле (2.5) объемов конечных тел пространства $\Lambda^{3}$ будем получать вещественные отрицательные значения. Этот факт особенно важен при измерении объемов тел, принадлежащих частично каждому из пространств $\widehat{H}^{3}$ и $\Lambda^{3}$. Скоординировать вычисления объемов таких тел можно по формуле

$$
V_{\Lambda^{3}}(F)=-V_{\widehat{H}^{3}}(F),
$$

где через $V_{\Lambda^{3}}(F)$ и $V_{\widehat{H}^{3}}(F)$ обозначен объем тела $F$ пространства $\mathbb{H}^{3}$, вычисленный в геометрии пространства $\Lambda^{3}$ и $\widehat{H}^{3}$ соттветственно. Доказательство этой формулы мы не приводим, поскольку в данной работе все вычисления проводим для конечных тел пространства $\widehat{H}^{3}$.

\section{3. ОРТОГОНАЛЬНАЯ КРИВОЛИНЕЙНАЯ СИСТЕМА КООРДИНАТ $C_{2, E}$}

3.1. Построение. Выберем в пространстве $\widehat{H}^{3}$ произвольный канонический репер $R^{*}=\left\{A_{1}, A_{2}, A_{3}, A_{4}, E\right\}$ первого типа, его координатную плоскость $A_{1} A_{2} A_{4}$ обозначим $\alpha$. Вершина $A_{4}$ репера $R^{*}$ расположена внутри абсолюта, поэтому $\alpha$ - расширенная гиперболическая плоскость. Пусть $E_{3}$ - ортогональная проекция точки $E$ на плоскость $\alpha$ : $E_{4}=A_{3} E \cap \alpha$ (Рис. 3.1). Прямой квазиугол (см. [17, с. 113]) плоскости $\alpha$ между прямыми $A_{1} A_{2}$ и $A_{1} A_{4}$, содержащий точку $E_{3}$, обозначим $\beta_{1}$. 
Прямую полуплоскость (см. там же) между прямыми $A_{1} A_{4}$ и $A_{2} A_{4}$, содержащую $E_{3}$, обозначим $\beta_{2}$. Пусть $\xi$ - прямой двугранный квазиугол (см. [6]) между плоскостями $\alpha$ и $A_{1} A_{2} A_{3}$, содержащий точку $E$.

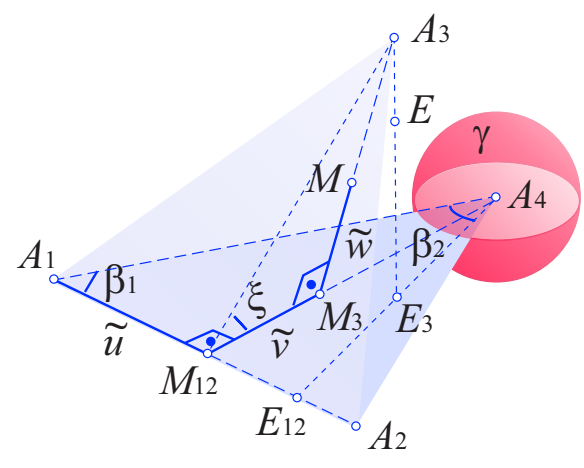

Рис. 3.1. Абсолют $\gamma$, система координат $C_{2, E}$

Набор фигур $\left\{A_{1}, A_{1} A_{2}, \alpha, \beta_{1}, \beta_{2}, \xi\right\}$ назовем эллиптической системой координат второго рода и обозначим $C_{2, E}$. Точку $A_{1}$, прямую $A_{1} A_{2}$ и плоскость $\alpha$ назовем соответственно началом, осъю и базой системы координат $C_{2, E}$. Точку $A_{3}$ назовем полюсом, углы $\beta_{1}, \beta_{2}, \xi-\kappa о о р д u$ натными углами этой системы.

Для произвольной точки $M$, лежащей во внешней области пространства $\widehat{H}^{3}$ относительно светового конуса точки $A_{3}$, введем следующие обозначения: $M_{3}$ - ортогональная проекция точки $M$ на плоскость $\alpha: M_{3}=A_{3} M \cap \alpha ; M_{12}$ - ортогональная проекция в плоскости $\alpha$ точки $M_{3}$ на прямую $A_{1} A_{2}: M_{12}=A_{4} M_{3} \cap A_{1} A_{2} ; \widetilde{u}$ - эллиптический отрезок между точками $A_{1}, M_{12}$, полностью или большей своей частью принадлежащий полуплоскости $\beta_{2} ; \widetilde{v}$ - гиперболический отрезок между точками $M_{3}, M_{12} ; \widetilde{w}$ - эллиптический отрезок между точками $M$, $M_{3}$, полностью или большей своей частью принадлежащий двугранному квазиуглу $\xi$.

Координатами точки $M$ в системе $C_{2, E}$ назовем тройку чисел

$$
u=\frac{|\widetilde{u}|}{\rho}, \quad v=\varepsilon \frac{|\widetilde{v}|}{\rho}, \quad w=\frac{|\widetilde{w}|}{\rho},
$$

где $\varepsilon=1$ ( $\varepsilon=-1)$, если точка $M_{3}$ принадлежит (не принадлежит) квазиуглу $\beta_{1}$. Координаты $(u, v, w)$ точки $M$ удовлетворяют условия:

$$
u \in[0, \pi), \quad v \in \mathbb{R}, \quad w \in[0, \pi) .
$$




\section{2. Связь между криволинейными и собственными координа-} тами точки. Пусть точка $M$, принадлежащая внешней области пространства $\widehat{H}^{3}$ относительно светового конуса точки $A_{3}$, задана в системе $C_{2, E}$ координатами $(u, v, w)$.

В репере $R^{*}=\left\{A_{1}, A_{2}, A_{3}, A_{4}, E\right\}$, присоединенном к системе $C_{2, E}$, точку $M$ зададим проективными координатами $\left(x_{1}: x_{2}: x_{3}: x_{4}\right)$ и собственными координатами $\left(\bar{x}_{1}: \bar{x}_{2}: \bar{x}_{3}: \bar{x}_{4}\right)$.

Тогда проективные координаты точек $M_{3}$ и $M_{12}$ в репере $R^{*}$ можно записать в виде $\left(x_{1}: x_{2}: 0: x_{4}\right)$ и $\left(x_{1}: x_{2}: 0: 0\right)$ соответственно.

По формулам $(1.2),(1.3)$ выразим длины отрезков $\widetilde{u}, \widetilde{v}$ и $\widetilde{w}$ через координаты точек $A_{1}, M, M_{3}, M_{12}$. С учетом обозначений (3.1) получаем:

$$
\begin{gathered}
\cos u= \pm \frac{x_{1}}{\sqrt{x_{1}^{2}+x_{2}^{2}}}, \quad \cosh v= \pm \frac{\sqrt{x_{1}^{2}+x_{2}^{2}}}{\sqrt{x_{1}^{2}+x_{2}^{2}-x_{4}^{2}}}, \\
\cos w= \pm \frac{\sqrt{x_{1}^{2}+x_{2}^{2}-x_{4}^{2}}}{\sqrt{x_{1}^{2}+x_{2}^{2}+x_{3}^{2}-x_{4}^{2}}} .
\end{gathered}
$$

Из выражений (3.2) находим зависимости между проективными координатами точки $M$ :

$$
\begin{gathered}
x_{2}^{2}=x_{1}^{2} \frac{\sin ^{2} u}{\cos ^{2} u}, \quad x_{4}^{2}=x_{1}^{2} \frac{\sinh ^{2} v}{\cos ^{2} u \cosh ^{2} v}, \\
x_{3}^{2}=x_{1}^{2} \frac{\sin ^{2} w}{\cos ^{2} u \cosh ^{2} v \cos ^{2} w} .
\end{gathered}
$$

Очевидно, что в репере $R^{*}$ условия (3.3) выполняются и для собственных координат точки $M$, определенных равенствами (2.1). Кроме того, собственные координаты подчинены условию (2.2).

Из системы требований (2.2), (3.3) находим выражения собственных координат точки $M$ в репере $R^{*}$ через ее криволинейные координаты в системе $C_{2, E}$ :

$$
\begin{array}{llrl}
\bar{x}_{1} & =\rho \cos u \cosh v \cos w, & \bar{x}_{2}=\rho \sin u \cosh v \cos w, \\
\bar{x}_{3}=\rho \sin w, & \bar{x}_{4}=\rho \sinh v \cos w,
\end{array}
$$

где $u \in[0, \pi), v \in \mathbb{R}, w \in[0, \pi)$.

\section{3. Элемент объема пространства $\widehat{H}^{3}$ в координатах системы} $C_{2, E}$. Пусть параметризация (3.4) определяет в репере $R^{*}$ собственные координаты точки $M$ из внешней относительно светового конуса точки 
$A_{3}$ области пространства $\widehat{H}^{3}$. Тогда точки $M_{u}, M_{v}$ и $M_{w}$ в репере $R^{*}$ заданы координатами:

$M_{u}(-\rho \sin u \cosh v \cos w: \rho \cos u \cosh v \cos w: 0: 0)$,

$M_{v}(\rho \cos u \sinh v \cos w: \rho \sin u \sinh v \cos w: 0: \rho \cosh v \cos w)$,

$M_{w}(-\rho \cos u \cosh v \sin w:-\rho \sin u \cosh v \sin w: \rho \cos w:-\rho \sinh v \sin w)$.

Вычисляя в параметризации (3.4) значения форм $\varphi$ и $\bar{\varphi}$ от координат точек $M_{u}, M_{v}, M_{w}$, получаем равенства

$$
\begin{gathered}
\gamma_{u u}=\rho^{2} \cosh ^{2} v \cos ^{2} w, \quad \gamma_{v v}=-\rho^{2} \cos ^{2} w, \quad \gamma_{w w}=\rho^{2}, \\
\gamma_{u v}=\gamma_{u w}=\gamma_{v w}=0,
\end{gathered}
$$

из которых следует, что система координат $C_{2, E}$ является ортогональной, а элемент объема пространства $\widehat{H}^{3}$ задан формулой

$$
d V=\rho^{3} \cosh v \cos ^{2} w d u d v d w
$$

Учитывая формулу (3.5) и ограничения на область задания системы $C_{2, E}$, объем $V$ тела $F$, заданного во внешней области относительно светового конуса точки $A_{3}$ областью $\bar{F}, \bar{F} \in \mathbb{R}^{3}$, найдем по формуле

$$
V=\rho^{3} \iiint_{\bar{F}} \cosh v \cos ^{2} w d u d v d w .
$$

4. ВЫЧИСЛЕНИЕ ОБЪЕМОВ ТЕЛ ПРОСТРАНСТВА $\widehat{H}^{3}$ В СИСТЕмЕ $C_{2, E}$

4.1. Основные определения. Выберем в пространстве $\widehat{H}^{3}$ расширенную гиперболическую плоскость $\alpha$ и ее полюс $A$ относительно абсолютной овальной поверхности $\gamma$. В плоскости $\alpha$ зададим конечную замкнутую двустороннюю линию $\sigma$. Линия $\sigma$ разбивает плоскость $\alpha$ на две компоненты, одна из которых гомеоморфна диску (или, на языке проективной геометрии, внутренней области проективной плоскости $\mathbb{P}^{2}$ относительно овальной линии). Назовем эту компоненту внутренностъю линии $\sigma$, ее замыкание обозначим $\sigma_{o}$. Коническая поверхность с вершиной $A$ и направляющей $\sigma$, образованная ортогональными к плоскости $\alpha$ эллиптическими прямыми, разбивает пространство $\widehat{H}^{3}$ на две компоненты. Рассмотрим ту из этих компонент, которая не содержит абсолют $\gamma$. Плоскость $\alpha$ разбивает эту компоненту на две конгруэнтные части, замыкание каждой из них назовем конечным ортогональным $h$ конусом с основанием $\sigma_{о}$ и вершиной $A$, указывая символом $h$ тип плоскости основания конуса. 
По определению, образующая конечного ортогонального $h$-конуса пространства $\widehat{H}^{3}$ равна половине эллиптической прямой, следовательно, ее длина равна $\pi \rho / 2$ (см., например, [17, п. 4.4.1]), где $\rho$ - радиус кривизны пространства $\widehat{H}^{3}$.

Если область $\sigma_{o}-n$-реберник, т. е. двусторонний многоугольник с ограниченной им областью плоскости [22], то ортогональный $h$-конус является конечной ортогоналъной $h$-пирамидой.

Сферы пространства $\widehat{H}^{3}$ образуют четыре типа: гиперсферы с комплексными радиусами и центрами в идеальных точках; эллиптические сферы с вещественными эллиптическими радиусами и центрами в собственных точках пространства $\widehat{H}^{3}$; гиперболические сферы с вещественными гиперболическими радиусами и центрами в собственных точках; орисферь с центрами на абсолюте.

Гиперсферы, гиперболические сферы и орисферы проективно эквивалентны, они являются овальными поверхностями. Эллиптические сферы являются кольцевыми поверхностями [11], т. е. невырожденными поверхностями второго порядка сигнатуры 0, они сплошь покрыты прямолинейными образующими двух семейств. В евклидовом пространстве кольцевые поверхности представлены однополостными гиперболоидами и гиперболическими параболоидами. В пространстве $\widehat{H}^{3}$ радиуса кривизны $\rho, \rho \in \mathbb{R}_{+}$, эллиптические сферы могут быть определены метрически как множества точек этого пространства, удаленных от собственной точки, называемой центром сферы, на эллиптическое расстояние $r, r \in(0, \pi \rho / 2)$, называемое радиусом сферы. Абсолютную полярную плоскость центра эллиптической сферы назовем базой этой сферы. Поскольку эллиптическое расстояние от собственной точки пространства $\widehat{H}^{3}$ до ее абсолютной полярной плоскости равно $\pi \rho / 2$, эллиптическая сфера радиуса $r$ является эквидистантной поверхностью высоты $\pi \rho / 2-r$.

Эллиптическая сфера с центром $A$ рассекает ортогональный $h$-конус $F$ с основанием $\sigma_{o}$ и вершиной $A$ на две связные компоненты. Ту компоненту, которая содержит основание $\sigma_{o}$ (вершину $A$ ), назовем $c \oint e$ рическим h-бочонком (сферическим $h$-сектором) $h$-конуса $F$. Высоту (радиус) секущей эллиптической сферы назовем высотой (радиусом) сферического $h$-бочонка ( $h$-сектора).

4.2. Объемы тел, ограниченных ортогональным $h$-конусом. Установим зависимость объема конечного ортогонального $h$-конуса пространства $\widehat{H}^{3}$ от площади его основания. Для этого рассмотрим конечный ортогональный $h$-конус $F$ с вершиной $A$ и основанием $\sigma_{o}$ в плоскости $\alpha$. 
Все образующие конуса $F$ принадлежат внешней относительно светового конуса точки $A$ области пространства $\widehat{H}^{3}$. Выберем на этой области координатную систему $C_{2, E}$ с полюсом в точке $A$ и базой $\alpha$. Пусть область $\sigma_{o}$ задана в системе $C_{2, E}$ числовой областью $\bar{S}$ изменения параметров $u, v$. Полагая в формулах (3.4) $w=0$, вычислим (см. [24]) площадь $S$ области $\sigma_{o}$ :

$$
S=\rho^{2} \iint_{\bar{S}} \cosh v d u d v .
$$

Для области $\overline{F_{k}}$, определяющей в системе $C_{2, E}$ сферический $h$-бочонок $F_{k}$ с основанием $\sigma_{o}$ и высотой $m$, выполняются условия: $(u, v) \in \bar{S}$, $w \in[0, m / \rho]$. Следовательно,

$$
\begin{aligned}
V\left(F_{k}\right) & =\rho^{3} \iiint_{\frac{F_{k}}{}} \cosh v \cos ^{2} w d u d v d w= \\
& =\rho S \int_{0}^{m / \rho} \cos ^{2} w d w=\frac{\rho S}{4}\left(\frac{2 m}{\rho}+\sin \frac{2 m}{\rho}\right) .
\end{aligned}
$$

По формуле (4.1) при $m=\pi \rho / 2$ получим объем $V(F)$ ортогонального $h$-конуса. Объем сферического $h$-сектора $F_{s}$ радиуса $r=\pi \rho / 2-m$ найдем по формуле: $V\left(F_{s}\right)=V(F)-V\left(F_{k}\right)$.

Итак, доказана следующая теорема.

Теорема 4.3. В пространстве $\widehat{H}^{3}$ радиуса кривизнь $\rho, \rho \in \mathbb{R}_{+}$, обгемы ортогонального $h$-конуса $F$, сферического $h$-бочонка $F_{k}$ высотой $m$ и сферического сектора $F_{s}$ радиуса $r$ могут быть вычислены соответственно по формулам

$$
\begin{gathered}
V(F)=\frac{\pi \rho S}{4}, \quad V\left(F_{k}\right)=\frac{\rho S}{4}\left(\frac{2 m}{\rho}+\sin \frac{2 m}{\rho}\right), \\
V\left(F_{s}\right)=\frac{\rho S}{4}\left(\frac{\pi \rho-2 r}{\rho}-\sin \frac{2 r}{\rho}\right) .
\end{gathered}
$$

Первая формула из (4.2) позволяет, в частности, вычислить объем конечной ортогональной $h$-пирамиды. Например, согласно Теореме 3.1 из [5] и Теореме 3 из [20] справедлива следующая теорема.

Теорема 4.4. В пространстве $\widehat{H}^{3}$ радиуса кривизны $\rho, \rho \in \mathbb{R}_{+}$, обгем $V$ конечной ортогональной $h$-пирамидъ с мерами $\widehat{A}_{1}, \ldots, \widehat{A}_{q}$ плоских 
углов основания может быть вычислен по формуле

$$
V=\frac{\pi \rho^{3}}{4}\left(\sum_{j=1}^{p} \widehat{A}_{j}-i \pi(p-2)\right)
$$

Если в основании конечной ортогональной $h$-пирамиды лежит прямоугольный трехреберник с длиной $a(b)$ эллиптического (гиперболического) катета, то ее обгем $V$ может быть вычислен по бормуле

$$
V=\frac{\pi \rho^{3}}{4} \ln \frac{\sinh \frac{b}{\rho}+\cosh \frac{b}{\rho} \sin \frac{a}{\rho}}{\sin \frac{a}{\rho}+\cos \frac{a}{\rho} \sinh \frac{b}{\rho}} .
$$

При вычислении в пространстве $\widehat{H}^{3}$ объемов пирамид с гиперболическим основанием могут быть полезны формулы площадей $n$-реберников различных видов в гиперболической плоскости положительной кривизны из работ [20], [19], [21], [12].

\section{ЛиТЕРАТУРА}

[1] J. Bolyai. Appendix. The Theory of Space Janos Bolyai (F. Kárteszi ed.). Budapest : Akadémiai Kiadó, 1987.

[2] R. Kellerhals. On the volume of hyperbolic polyhedra. Math. Ann., 285:541-569, 1989.

[3] J. H. Lambert. Die Theorie der Parallellinien. Leipziger : Leipziger Magazin für Reine und Angewandte Mathematik, 1786.

[4] J. Murakami, A. Ushijima. A volume formula for hyperbolic tetrahedral in terms of edge lengths. Journal of Geometry, 83(1-2):153-163, 2005.

[5] L. N. Romakina. The area of a generalized polygon without parabolic edges of a hyperbolic plane of positive curvature. Asian Journal of Mathematics and Computer, 10(4):293-310, 2016.

[6] L. N. Romakina. Dihedrons of a hyperbolic three-space of positive curvature. International Electronic Journal of Geometry, 9(2):50-58, 2016.

[7] L. Schläfli. Theorie der vielfachen Kontinuität. Gesammelte mathematishe Abhandlungen. Basel : Birkhauser, 1950.

[8] Н. В. Абросимов. Об объемах многогранников в пространствах постоянной кривизны. Вестник Кем. ун-та, 3(1):7-13, 2011.

[9] М. П. Замаховский Б. А. Розенфельд. Геометрия групn Ли. Симметрические, параболические и периодические пространства. М. : МЦНМО, 2003.

[10] Е. В. Винберг. Объемы неевклидовых многогранников. Успехи мат. наук, 48(2(290)):17-46, 1993.

[11] Н. В. Ефимов. Высшая геометрия. М. : Наука, 1971.

[12] В. А. Клычкова. Трипрямоугольник гиперболической плоскости положительной кривизны. Сб. статей Междунар. научно-практ. конф.., pages 14-17, 2016.

[13] Н. И. Лобачевский. Воображаемая геометрия. Учен. зап. Казан. ун-та, 1:3-88, 1835.

[14] Б. А. Розенфельд. Неевклидовы пространства. М. : Наука, 1969.

[15] Л. Н. Ромакина. Геометрии коевклидовой и копсевдоевклидовой плоскостей. Саратов : Научная книга, 2008. 
[16] Л. Н. Ромакина. Аналоги формулы Лобачевского для угла параллельности на гиперболической плоскости положительной кривизны. Сиб. электрон. матем. изв., 10:393-407, 2013.

[17] Л. Н. Ромакина. Геометрия гиперболической плоскости положительной кривизны : в 4 частях. Ч. 1: Тригонометрия. Саратов : Изд-во Сарат. ун-та, 2013.

[18] Л. Н. Ромакина. Геометрия гиперболической плоскости положительной кривизны : в 4 частях. Ч. 2: Преобразования и простые разбиения. Саратов : Изд-во Сарат. ун-та, 2013.

[19] Л. Н. Ромакина. О площади эллиптического четырехугольника Саккери на гиперболической плоскости положительной кривизны. Математика. Механика, 15:6669, 2013.

[20] Л. Н. Ромакина. Теорема о площади прямоугольного трехреберника гиперболической плоскости положительной кривизны. Дальневост. матем. журн., 13(1):127147, 2013.

[21] Л. Н. Ромакина. Аналоги формулы Герона для трехреберников типов еее $(i)$, еее(iii) гиперболической плоскости положительной кривизны. Математика. Meханика, 17:52-55, 2015.

[22] Л. Н. Ромакина. Классификация тетраэдров с негиперболическими гранями в гиперболическом пространстве положительной кривизны. Чебышевский сб., 16(2):208-221, 2015.

[23] Л.Н.Ромакина. Развитие представлений о геометрии окружающего пространства. Евразийское научное объединение, 1(10):18-21, 2015.

[24] Л. Н. Ромакина. К теории площадей гиперболической плоскости положительной кривизны. Publ. Inst. Math. (Beograd) (N.S.), 99(113):139-154, 2016.

[25] И. Х. Сабитов. Алгебраические методы решения многогранников. Успехи мат. наук, 66(3):445-505, 2011.

Поступила в редакиию 26 мая 2017, принята к печати 17 августа 2017.

Людмила Николаевна Ромакина

САРАТОВСКИЙ ГОСУДАРСТВЕННЫЙ УНИВЕРСИТЕт, РосСИЯ

Email: romakinaln@mail.ru

ORCID: orcid.org/0000-0002-3695-2076 